\title{
How Question Characteristics Impact Answer Outcomes on Social Question-and-Answer Websites
}

\author{
Yiming Zhao, Wuhan University, Wuhan, China \\ (iD) https://orcid.org/0000-0001-8182-456X \\ Linrong $\mathrm{Wu}$, Wuhan University, Wuhan, China \\ (iD) https://orcid.org/0000-0002-3810-1179 \\ Jin Zhang, University of Wisconsin-Milwaukee, USA \\ (iD https://orcid.org/0000-0002-6665-6606 \\ Taowen Le, Weber State University, USA \\ (iD) https://orcid.org/0000-0001-5545-6142
}

\begin{abstract}
Inducing more and higher-quality answers to questions is essential to sustainable development of social question-and-answer (SQA) websites. Previous research has studied factors affecting question success and user motivation in answering questions, but how a question's own characteristics affect the question's answer outcome on SQA websites remains unknown. This study examines the impact of the characteristics of a question, namely readability, emotionality, additional descriptions, and question type, on the question's answer outcome as measured by number of answers, average answer length, and number of "likes" received by answers to the question. Regression analyses reveal that readability, additional descriptions, and question type have significant impact on multiple measurements of answer outcome, while emotionality only affects the average answer length. This study provides insights to SQA website builders as they instruct users on question construction. It also provides insights to SQA website users on how to induce more and higher-quality answers to their questions.
\end{abstract}

\section{KEYWORDS}

Answer Outcome, Community Question Answering, Information Management, Question Characteristics, Social Q\&A

\section{INTRODUCTION}

As the Internet continues to impact people and societies (Teo, 2007; Lane, et al., 2017), researchers have explored ways to maximize successes of Internet-based technology implementations or global information management (Roztocki \& Weistroffer, 2011; Caprio, et al., 2015; Hung, et al., 2016; Silic 
\& Back, 2016; Soja, 2016; Chatterjee, et al., 2017). Social question-and-answer (SQA) websites are an important category of such implementations.

An SQA website is an online community in which users can post or answer questions. Users of these websites seek answers to a wide variety of questions that are organized under topical categories; they also share their own knowledge, experiences, and opinions (Zhang et al., 2020; Oh, 2018). Thanks to the extensive participation of Web users, SQA websites have become some of the most popular destinations for online information seekers (Zhao et al., 2018; Oh, 2018; Jiang et al., 2018; Zhang \& Zhao, 2013). SQA websites such as Yahoo!Answers, Stack Overflow, and Answers.com have been listed among the top 50 popular websites in the reference category of Alexa (Alexa, 2020).

Inducing more and higher-quality answers is essential to sustainable development of SQA websites. To facilitate sustainable development of SQA websites, numerous researchers have studied user motivation in knowledge contribution (Wasko \& Faraj, 2000), factors attracting or motivating answerers (Jin et al., 2015), criteria measuring question success or failure (Shah et al., 2012), searchresult relevance judgment (Zhang et al., 2020), search-result measurement (Zhang et al., 2019), answer quality (Zhao et al., 2019), website evaluation (Huang et al., 2006), and solutions to improve response rate (Liu \& Jansen,2017; Oh, 2018; Elalfy et al., 2017; Ren et al., 2018; Sin et al., 2018; Wang, et al., 2018; Li et al., 2016). However, little is known about how the question itself affects the answer outcome on an SQA website.

To bridge this gap, this study aims to reveal impact of question characteristics (readability, emotionality, additional descriptions, and question type) on answer outcome. Research questions include (1) whether question readability affects its answer outcome on SQA websites, (2) whether question emotionality affects its answer outcome on SQA websites, (3) whether additional descriptions in a question affect its answer outcome on SQA websites, and (4) whether question type affects its answer outcome on SQA websites.

The contributions of this paper are summarized as follow:

- It adds to knowledge about question evaluation; specifically, it sheds light on how to induce more and higher-quality answers on SQA websites by constructing better questions.

- It uses more-refined metrics, namely, numbers of answers, average answer length, and number of "likes" received by answers, to measure answer outcome of a question. In contrast, prior studies measured question success primarily by judging whether a question was answered or not.

- It provides insights to SQA website builders as they instruct users on question construction. It also provides insights to SQA website users on how to attract more and higher-quality answers to their questions.

\section{RELATED RESEARCH}

\subsection{Factors Impacting Knowledge-Contribution Behavior on SQA Websites}

User motivation is a key determinant of whether questions are answered or whether they receive high-quality answers (Chua\& Banerjee, 2015). Previous studies have examined internal and external motivation factors to uncover mechanisms triggering user knowledge-contribution behavior (Casalo et al., 2010; Chen, 2007).

Primary internal factors motivating users to contribute knowledge in virtual communities include individual characteristics and levels of openness (Cabrera \& Cabrera, 2005), confidence to spread knowledge (Wasko \& Faraj, 2005), satisfaction, self-efficacy, outcome expectations, and trust (Choudhury et al., 2014). Additionally, self-enjoyment, learning, personal income, empathy, social participation, and reciprocity have also been found to have influence on users' knowledgecontribution behavior (Oh, 2012). 
Primary external factors motivating users to contribute knowledge in virtual communities include community culture, management support, incentive mechanism, and organizational structure. Jeon et al. (2006) investigated the performance of a price-based question-and-answer system in their study of Google's answers. They found that providing higher answer prices led to longer answers rather than better answers. In the study by Harper et al. (2010), the answer quality of the Google Answers platform was generally higher than that of the free SQA websites they investigated, and the answer quality increased as the value added to the question increased.

In the context of SQA, Jin et al. (2015) concluded that members' self-presentation, other members' recognition, and social learning opportunity positively influenced users' knowledge-contribution behavior. To predict active knowledge sharing on SQA websites, Liu and Jansen (2017) proposed a model based on user profile, posting behavior, language style, and social activity.

\subsection{Answer Outcome and its Influencing Factors in the Context of SQA Websites}

Tens of thousands of questions are posted everyday on SQA websites, some generating active discussions while others remain unanswered. Shah, Radford, and Connaway (2012) explored possible reasons why certain questions remain unanswered on Yahoo! Answers and discovered that ambiguity, complexity, inappropriateness, and multiplicity of the question text affected question success, and that questions with high ambiguity and structural complexity often experienced greater difficulty in attracting answers. Choi et al. (2013) extracted a variety of textual features based on 200 unanswered questions and 200 answered questions on Yahoo!Answers and found that the level of clarity, presence of taboo words, punctuation, and presence of additional information were the most significant factors in their predictive model of answer outcome. They also studied the impact of identity information of health-related questions on answer outcome and discovered that disclosing appropriate amount and type of identity information in a question would generate more interactions. Teevan et al. (2011) suggested that the likelihood of quickly receiving answers could be increased by ending a statement of information needs with a question mark and explicitly scoping the audience.

Answer quality is also a measure of answer outcome. In Adamic and associates' study (Adamic et al., 2008), answer length, number of answers, and users' history records were important measurements of best answers. While this particular research treated longer answers as better answers, another research (Kim and Oh, 2009) suggested that users were more inclined to provide short and straightforward answers.

Shah and Pomerantz (2010) recruited workers on Amazon Mechanical Turk to assess answer quality on Yahoo!Answers. They extracted multiple features from question texts and answers and trained a variety of classifiers to select the best answers. They found that the overall best answers they picked well matched the "best answers" as selected by the questioner. Jeon et al. (2006) proposed a framework to predict answer quality using non-textual features. They extracted 13 non-textual features from the Q\&A pair data on Naver Q\&A Service, including answerer's acceptance ratio, questioner's self- evaluation, answerer's activity level, and answerer's category specialty.

In summary, existing studies have focused more on internal and external factors motivating users to answer questions, and few have explored the impact of the characteristics of a question itself on its answer outcome. The few studies that have studied answer outcome and its influencing factors have used the presence of answers as the primary success indicator. In contrast, we investigate potential impact of question characteristics on answer outcome in terms of number of answers, average answer length, and average number of "likes" received by answers. 


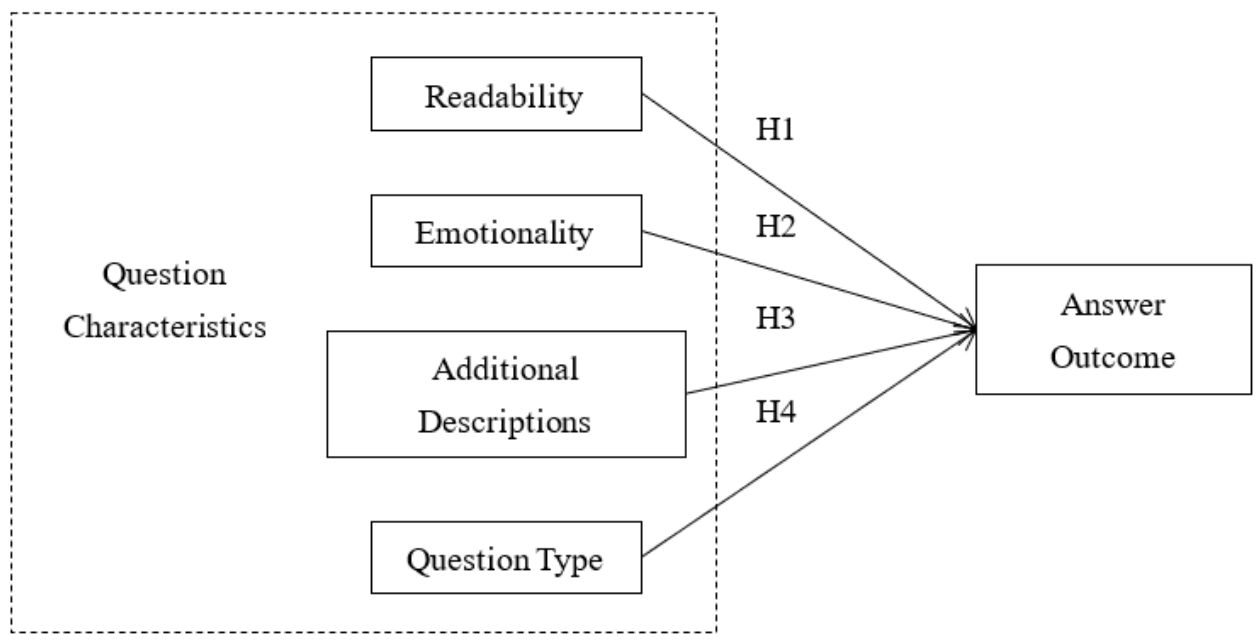

\section{RESEARCH MODEL AND HYPOTHESES}

\subsection{Research Model}

As shown in Figure 1 our research model includes four question characteristics as independent variables: text readability, text emotionality, additional descriptions, and question type. The dependent variable is answer outcome. The definitions of the relevant variables are summarized in Table 1.

This paper measures answer outcome in terms of both "quantity" and "quality". The quantity aspect is measured by the total number of answers a question attracts, and the quality aspect is measured by the average answer length and the average number of "likes" received by those answers.

A longer answer indicates a greater willingness to help the questioner, and a longer answer contains more information. Harper et al. (2008) concluded that answer length was an indicator of answer quality on SQA websites. Agichtein et al. (2008) also suggested that answer length was an

Table 1. Definitions of Independent Variables

\begin{tabular}{|l|l|}
\hline \multicolumn{1}{|c|}{ Variable } & \multicolumn{1}{c|}{ Definition } \\
\hline Text Readability & The easiness of text; the extent to which readers understand the text \\
\hline Text Emotionality & The social-emotional nature of what is contained in the text \\
\hline Additional Descriptions & $\begin{array}{l}\text { Additional paragraphs attached to the question to provide detailed information that helps } \\
\text { readers better understand the information needs }\end{array}$ \\
\hline Question Type & Whether a question is informational or conversational \\
\hline Answer Outcome & $\begin{array}{l}\text { Both quantity and quality aspects of answer outcome are examined in this study. Specifically, } \\
\text { answer outcome is measured using three metrics: number of answers to a question, average } \\
\text { answer length, and average number of "likes" received by answers to a question. }\end{array}$ \\
\hline
\end{tabular}

important criterion in evaluating answer quality on Yahoo!Answers. 
Answer quality can also be reflected by the number of "likes" received by a question's answers. On an SQA website, users may agree or disagree with others; greater number of "likes" suggests answers receiving greater approval and hence being of higher quality. For example, Li et al. (2015) used the number of "likes" received by answers as an indicator of answer quality.

As a particular question might generate multiple answers, this paper examines the overall answer outcome. Therefore, it uses average length of answers and average number of "likes" as measurements of answer quality.

\subsection{Text Readability and Answer Outcome}

Text readability refers to the extent to which readers can understand and comprehend the text (Klare, 1974). Several previous studies have employed this indicator. For example, Choi et al. (2013) used question readability in their model to predict question success.

Theoretically, readable questions present contents clearly and therefore should be more inviting to readers. Moreover, readers understand questions of high readability more thoroughly and would be able to provide better answers. Therefore, for questions of high readability, we expect that the number of answers obtained would be greater and that the answer quality would be higher.

Hence, we propose the following hypotheses:

HIa: The more readable the question text, the greater the number of answers the question obtains. $H 1 b$ : The more readable the question text, the longer the average length of answers the question obtains. $H 1 c$ : The more readable the question text, the higher the average number of "likes" its answers obtain.

\subsection{Question Emotionality and Answer Outcome}

People posting questions on SQA websites may express emotions in the context of their questions, such as joy, anger, sadness, happiness, or other sentiments. Kim and Oh (2009) observed that SQA websites were interactive platforms with social elements, where people could exchange emotions such as disappointment and gratitude. When a question contains words reflecting the questioner's emotions, the questioner's emotions may have an impact on the reader of the question (Buntrock, 2007).

In a previous study, Harper et al. (2008) used "expression of gratitude" in their answer-quality prediction model and investigated its impact on answer quality.

Theoretically, when a question contains words of emotions, it would more likely generate resonance and compassion in readers, motivating their participation in related discussions and responses to the question. Moreover, emotionally expressive questions tend to invoke empathy or sympathy in the respondent, driving the respondent to provide higher-quality answers to help the questioner. Thus, we expect questions with emotionality to attract more and better answers.

Hence, we propose the following hypotheses:

$H 2 a$ : Questions with emotionality generate more answers than questions without emotionality.

$H 2 b$ : On Average, questions with emotionality receive longer answers than questions without emotionality.

$H 2 c$ : On average, answers to questions with emotionality receive more "likes" than answers to questions without emotionality.

\subsection{Additional Descriptions and Answer Outcome}

To a complex question, the questioner may add additional detail information such as a paragraph of text to explain the content of the question. The presence of such additional information may have an impact on question answers. Saha et al. (2013) noticed that questions that were too short or too lengthy had difficulties attracting answers. Asaduzzaman et al. (2013) found that question text 
hindered answer generation when expressed vaguely and roughly. Choi et al. (2013) concluded that the availability of detail information contributed to question success.

Theoretically, detailed supplementary information in a question may motivate the respondent to answer the question. Moreover, such additional descriptions would help user better understand the question, thereby enabling the respondent to provide better answers.

Hence, we propose the following hypotheses:

H3a: Questions with additional descriptions receive more answers than questions without additional descriptions.

$H 3 b$ : On average, questions with additional descriptions receive longer answers than questions without additional descriptions.

$H 3 c$ : On average, answers to questions with additional descriptions receive more "likes" than answers to questions without additional descriptions.

\subsection{Question Type and Answer Outcome}

Questions can be categorized into different types depending on how they are presented. Different types of questions could generate different answer outcome. Harper et al. (2009) classified questions into two categories: informational questions and conversational questions.

An informational question aims to obtain factual knowledge or suggestions to solve a practical problem. The answers are relatively professional, uniform, and less debatable. A specific example of informational questions might be "Does prolonged sedentary activity lead to obesity?" A conversational question aims to obtain different perspectives or opinions from different respondents. Answers to conversational questions tend to be less structured. A sample question might be "Which do you think is better, Coca-Cola or Pepsi?" Harper et al. (2009) found that informational questions and conversational questions differed significantly in the use of words and sentences.

Theoretically, one would expect that conversational questions bring more answers because they promote discussions, while informational questions do not produce too many answers because of the limited possibilities of correct answers. Moreover, one would expect answer quality for informational questions to be higher than for conversational questions because answers to informational questions are more definite and more practically useful while answers to conversational questions are mostly subjective opinions.

Hence, we propose the following hypotheses:

H4a: Informational questions generate fewer answers than conversational questions.

$H 4 b$ : On average, informational questions generate longer answers than conversational questions.

$H 4 c$ : On average, answers to informational questions receive more "likes" than answers to conversational questions.

\section{METHODOLOGY}

\subsection{Data Collection}

With over 60 million monthly hits, Yahoo!Answers is the most popular SQA website in the world (Gazan, 2014). This study employed Python crawler in collecting question texts and answers from Yahoo!Answers. The data collected included questions, additional descriptions of questions, question categories, posting times, numbers of answers, actual answer content, and number of "likes" received by each answer. Table 2 describes the data fields with illustrating examples. The actual data collection took place on February 21, 2019. Data on Yahoo!Answers with a time stamp between January 21 and February 21, 2019 was collected. 
Table 2. Description of Data Fields

\begin{tabular}{|l|l|l|}
\hline \multicolumn{1}{|c|}{ Data Field } & \multicolumn{1}{c|}{ Description } & \multicolumn{1}{c|}{ Example } \\
\hline Question & The actual wording of a question & $\begin{array}{l}\text { Do you think that the Kent State shooting } \\
\text { was justified rightly? }\end{array}$ \\
\hline Additional Description & Supplementary details for a question & 4 died and 9 were injured \\
\hline Question Category & $\begin{array}{l}\text { Subject area to which a question } \\
\text { belongs }\end{array}$ & Arts \& Humanities \\
\hline Posting Time & $\begin{array}{l}\text { Year, month, day, hour, minute, and } \\
\text { second when a question was posted } \\
\text { on the website }\end{array}$ & 20190126181443 \\
\hline Number of Answers & $\begin{array}{l}\text { Number of responses to a posted } \\
\text { question }\end{array}$ & 43 \\
\hline Like Count & $\begin{array}{l}\text { Total number of "likes" received by } \\
\text { answers to a posted question }\end{array}$ & 163 \\
\hline
\end{tabular}

Figure 2. Days after a Question Was Posted vs. Rate of Change in Numbers of Answers

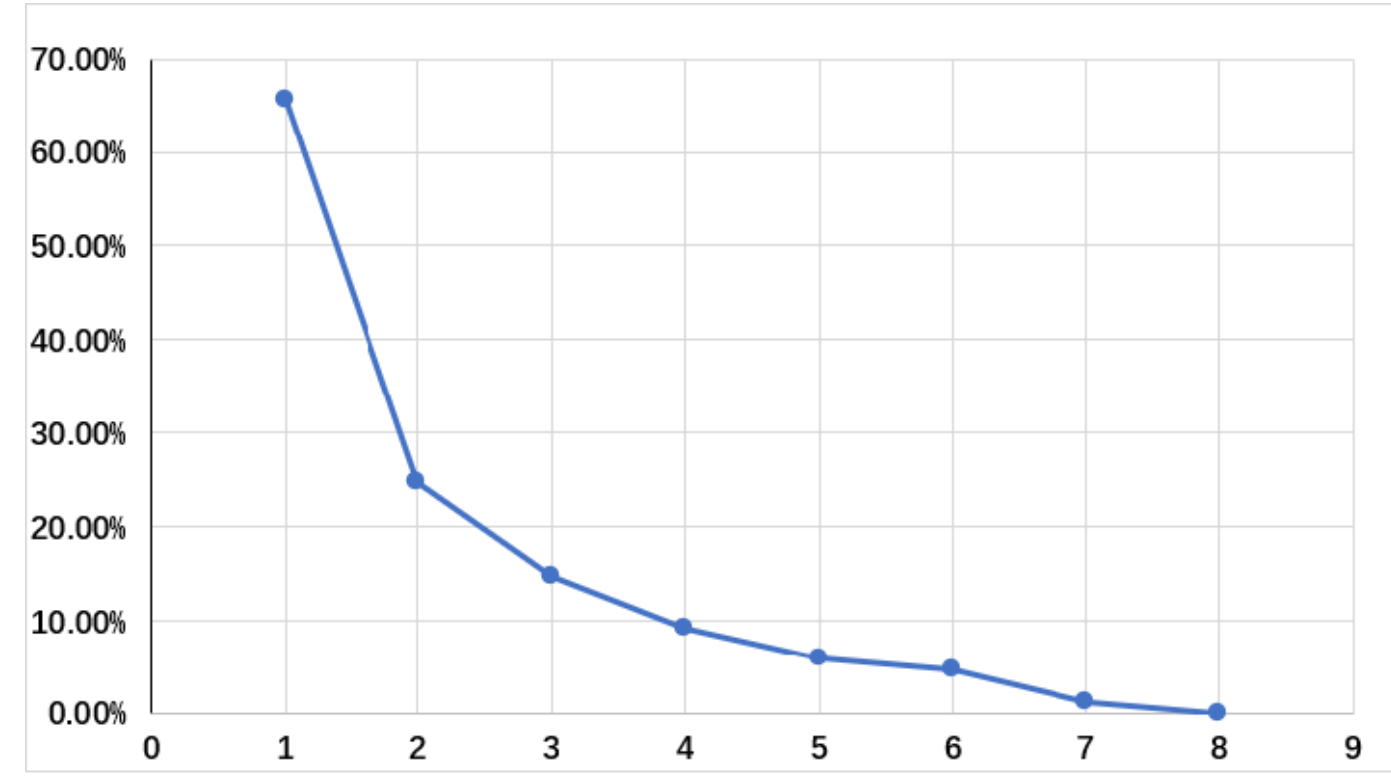

The number of answers is an important criterion in this study; however, on SQA websites such as Yahoo!Answers, the number of answers to each question may change over time. To assess the potential impact of such changes, we conducted a pre-experiment, monitoring the questions and corresponding answers on Yahoo!Answers day by day to track changes in numbers of answers to the relevant questions. The results are shown in Figure 2.

Based on the findings of the pre-experiment, changes in numbers of answers occur the most on the first three days after the initial posting of the questions, especially on the first day. After that, changes become progressively infrequent. By the fourth day, the change rate dropped to less than $10 \%$. Therefore, this study only included questions posted at least four days prior to the crawler's crawling time. After excluding data within four days of the crawler's crawling time, 20110 questions along 
with their corresponding answers, numbers of "likes" received by their answers, and other relevant data were collected for further analysis.

\subsection{Measurements of Variables}

Based on the research model and hypotheses, this study examines four independent variables pertaining to questions and three dependent variables pertaining to answer outcome. The measurement of each variable is explained in the following sections.

\subsubsection{Question Readability}

Many factors can influence the readability of a text, such as its average sentence length, the number of unfamiliar words, and the complexity of grammar used. Consequently, there are different ways to measure text readability. Commonly-used measurements include Flesch Reading Ease (Flesch, 1948), Flesch-Kincaid Grade Level (Kincaid, 1992), Gunning Fog Index (Gunning, 1968), SMOG Index (McLaughlin, 1969), and Automated Readability Index (ARI) (Smith \& Senter, 1967).

Indices such as Flesch Reading Ease, Flesch-Kincaid Grade Level, and Gunning Fog Index use the average number of syllables per word to measure word difficulty, which is considered unreliable in some cases (Scott, 2019). SMOG Index is suitable for texts with more than 30 sentences. On the other hand, ARI uses the number of characters per word to represent word difficulty, has no limitation pertaining to the number of sentences, and hence is more accurate and suitable for this study. Therefore, this study uses ARI to measure question readability.

The specific calculation of the $A R I$ indicator is described in Equation (1). The value output of the $A R I$ calculation approximately represents the level of education required to understand the text.

$$
A R I=4.71 \times\left(\frac{\text { characters }}{\text { words }}\right)+0.5 \times\left(\frac{\text { words }}{\text { sentences }}\right)-21.43
$$

In Equation (1), characters, words, and sentences represent the number of letters, words, and sentences in the text, respectively. The larger the value of the ARI calculation result, the more difficult it is to understand the text, and the less readable the text is. For example, when the value is 12 , it means that reading this text requires a comprehension level of a 17-year-old high-school sophomore in the United States.

To facilitate statistical analysis, we normalize the results of the $A R I$ calculation and use the difference between the normalized value and 1 as the final readability value. After such processing, the readability is distributed in the $[0,1]$ range, and the larger the value, the higher the readability, and the less difficult to understand the question text.

\subsubsection{Question Emotionality}

To determine whether a given question is emotional, this study uses text processing tool TextBlob (Steven, 2019) for sentiment measurement of question texts. TextBlob is an open source toolkit written in Python and can be used to perform natural-language processing tasks such as part-of-speech tagging, noun component extraction, sentiment analysis, and text translation. TextBlob analyzes a sentence and returns a sentiment value in the interval of $[-1,1]$. Since this study examines emotionality of an entire question and a question might contain multiple sentences, multiple sentiment values might be provided for one question. If there is a non-zero value, the question contains emotionality; if all sentiment values are zeroes, the question is of no emotionality.

Therefore, question emotionality is regarded as a binary variable; questions having emotionality are labeled with " 1 ", and questions having no emotionality are labeled with " 0 ". 


\subsubsection{Additional Descriptions}

Yahoo!Answers limits each question to 140 characters but allows 1500 additional characters for supplemental information. Therefore, additional information can be entered in the question detail box as clarification about the question posted.

In this study, additional descriptions are also regarded as a binary variable; questions with additional descriptions are labeled with " 1 ", and questions without additional descriptions are labeled with " 0 ".

\subsubsection{Question Type}

Following the classification of Harper et al. (2009), this study divides questions into two categories: informational and conversational.

We used a machine-learning approach to classify the 20110 questions. We employed a Bayesian model and selected a dataset of annotated questions openly available to researchers at Yahoo Labs (Yahoo Research, 2019) as the training data. The dataset consisted of 5,025 randomly-selected questions from Yahoo!Answers. 2228 of them were informational questions and were labeled with " 0 "; 1784 of them were conversational questions and were labeled with " 1 "; 1013 of them were disputed and labeled with " 2 ". We removed the disputed data and obtained a total of 4012 questions as the final training dataset to train the model.

This paper uses the trained model to classify the question texts to be analyzed, dividing the 20110 questions into 10,130 informational and 9980 conversational questions. Given the nature of the data, question type is also regarded as a binary variable; informational questions are labeled with " 0 " and conversational questions with " 1 ".

\subsubsection{Answer Outcome}

Answer outcome in this study is measured by three metrics: number of answers to a question, average length of answers to a question, and average number of "likes" received by answers to a question.

Number of answers is the total number of valid responses from other users to a question. It represents the "quantity" aspect of answer outcome.

Average length of answers refers to the average number of words contained in answers to a particular question. It is believed that the word count of an answer reflects the respondent's degree of conscientiousness, and conscientious respondents often write more words to increase the "quality" of their answers. Since a question often receives multiple answers of varying lengths, this paper uses the total number of words divided by the number of answers to calculate the average length of answers as an indicator of the "quality" aspect of answer outcome.

Average number of "likes" received by answers to a question is calculated by totaling "likes" received by all answers to the question and dividing the total by the number of answers to the question. It is another indicator of the "quality" aspect of answer outcome.

\subsection{Regression Analyses}

This paper explores the impact of four question characteristics (independent variables) on answer outcome as measured by three metrics (dependent variables). Since Multiple Linear Regression model can be used to analyze correlations between one dependent variable and multiple independent variables, this study utilizes the Multiple Linear Regression model to analyze the research data, examining how multiple independent variables are related to one dependent variable (Kenton, 2019).

The mathematical model is shown in Equation (2).

$y=\beta_{0}+\beta_{1} x_{1}+\cdots+\beta_{p} x_{p}+\varepsilon$ 
Table 3. Descriptive Statistics for Each Variable

\begin{tabular}{|c|c|c|c|c|c|c|c|}
\hline \multicolumn{2}{|c|}{ Name of Variable } & $N$ & $\begin{array}{l}\text { Min. } \\
\text { Value }\end{array}$ & $\begin{array}{l}\text { Max. } \\
\text { Value }\end{array}$ & Mean & Std. Dev. & Variance \\
\hline \multicolumn{2}{|c|}{ Readability } & 20110 & 0 & 1 & 0.548 & 0.185 & 0.034 \\
\hline \multicolumn{2}{|c|}{ Emotionality } & 20110 & - & - & - & - & - \\
\hline \multicolumn{2}{|c|}{ Additional Descriptions } & 20110 & - & - & - & - & - \\
\hline \multicolumn{2}{|c|}{ Question Type } & 20110 & - & - & - & - & - \\
\hline \multirow{3}{*}{$\begin{array}{l}\text { Answer } \\
\text { Outcome }\end{array}$} & $\begin{array}{l}\text { Number of } \\
\text { Answers }\end{array}$ & 20110 & 0 & 304 & 9.363 & 9.937 & 98.753 \\
\hline & $\begin{array}{l}\text { Average } \\
\text { Answer } \\
\text { Length }\end{array}$ & 20110 & 0 & 560.5 & 43.735 & 49.382 & 2438.605 \\
\hline & $\begin{array}{l}\text { Average } \\
\text { Number } \\
\text { of Likes } \\
\text { Received by } \\
\text { Answers }\end{array}$ & 20110 & 0 & 7.12 & 0.867 & 0.813 & 0.661 \\
\hline
\end{tabular}

In Equation (2), $y$ is the dependent variable, a random quantitative observation value; $x_{1}, \cdots x_{p}$ are independent variables; $\beta_{0}$ is the constant term, $\beta_{1}, \cdots \beta_{p}$ are partial regression coefficient. $\beta_{i}(i=1,2, \cdots p)$ represents the average amount of change in the dependent variable $y$ caused by each unit change of independent variable $x_{j}$ alone when the other independent variables are fixed. $\varepsilon$ is random error, also called residual error, which is the part of the change in y that cannot be explained by independent variables.

The multiple linear regression equation estimated by the sample is shown in Equation (3).

$\hat{y}=b_{0}+b_{1} x_{1}+\cdots+b_{p} x_{p}$

In Equation (3), $y$ is the average estimated value or average predicted value of the dependent variable $y$ when each $x$ takes a set of constant values. $b_{0}, b_{1}, \cdots b_{p}$ are the sample estimate values of $\beta_{0}, \beta_{1}, \cdots \beta_{p}$.

There are three dependent variables in the research model of this study. Therefore, three multiple linear regression analyses are conducted, and three multiple linear regression equations are obtained.

\section{RESULTS AND DISCUSSION}

\subsection{Descriptive Statistics}

The measured values of all variables in the research model of this study are first statistically analyzed using SPSS 22 software, and the descriptive statistical results are shown in Table 3.

The correlation matrix for the dependent variables is shown in the Table 4. 
Table 4. Correlation Matrix for the Dependent Variables

\begin{tabular}{|l|l|l|l|}
\hline & Number of Answers & Average Answer Length & $\begin{array}{c}\text { Average Number of Likes Received by } \\
\text { Answers }\end{array}$ \\
\hline Number of Answers & 1.000 & -.048 & .238 \\
\hline $\begin{array}{l}\text { Average Answer } \\
\text { Length }\end{array}$ & -.048 & 1.000 & -.003 \\
\hline $\begin{array}{l}\text { Average Number of } \\
\text { Likes Received by } \\
\text { Answers }\end{array}$ & .238 & -.003 & 1.000 \\
\hline
\end{tabular}

\subsection{Regression Analysis on Number of Answers}

The Multiple Linear Regression model pertaining to number of answers is shown in Equation (4), where $N O A$ stands for Number of Answers, $R E_{1}$ stands for Readability, $E M_{1}$ stands for Emotionality, $A D_{1}$ stands for Additional Descriptions, and $Q T_{1}$ stands for Question Type. The results are shown in Table 5.

$\hat{y}_{1}(\mathrm{NOA})=b_{10}+b_{11} \times \mathrm{RE}_{1}+b_{12} \times \mathrm{EM}_{1}+b_{13} \times \mathrm{AD}_{1}+b_{14} \times \mathrm{QT}_{1}$

The results in Table 5 indicate that this Multiple Linear Regression model pertaining to number of answers is statistically significant $(F=104.609, p<0.001)$. The results also show that three of the four independent variables in the research model of this study, readability, additional descriptions, and question type, have a statistically significant impact on number of answers $(p<0.05)$.

Question readability has a significant positive impact on number of answers (coefficient $=$ $2.847>0, p<=0.001)$. In other words, the higher the readability of a question, the more answers it receives, which validates Hypothesis Hla.

Question emotionality has no significant impact on number of answers $(p=0.967)$, thus indicating that Hypothesis $H 2 a$ is invalid.

Additional descriptions have a significant negative impact on number of answers (coefficient $=$ $-1.319<0, p<=0.001$ ), indicating that questions with additional descriptions receive less answers than questions without additional descriptions. Thus, the opposite of Hypothesis $H 3 a$ is confirmed.

Table 5. Regression Results with Number of Answers as Dependent Variable

\begin{tabular}{|l|l|l|l|l|}
\hline \multicolumn{1}{|c|}{ Name of Variables } & \multicolumn{1}{|c|}{ Coefficient } & \multicolumn{1}{c|}{$\begin{array}{c}\text { Std. } \\
\text { Deviation }\end{array}$} & \multicolumn{1}{|c|}{$T$-value } & \multicolumn{1}{c|}{ Significance } \\
\hline Readability & 2.847 & 0.377 & 7.552 & $0.000^{* * *}$ \\
\hline Emotionality & 0.006 & 0.140 & 0.041 & 0.967 \\
\hline Additional Descriptions & -1.319 & 0.139 & -9.488 & $0.000^{* * *}$ \\
\hline Question Type & 2.406 & 0.140 & 17.226 & $0.000^{* * *}$ \\
\hline Adjusted $R^{2}$ & 0.020 & & & \\
\hline Regression Model Significance & 0.000 & & & \\
\hline$F$-value & 104.609 & & & \\
\hline Note*** means $p<0.001 * *$ means $p<0.01 *{ }^{*}$ means $p<0.05$ & & & \\
\hline
\end{tabular}


Table 6. Regression Results with Average Answer Length as Dependent Variable

\begin{tabular}{|l|l|l|l|l|}
\hline \multicolumn{1}{|c|}{ Name of Variable } & \multicolumn{1}{c|}{ Coefficient } & \multicolumn{1}{c|}{$\begin{array}{c}\text { Std. } \\
\text { Deviation }\end{array}$} & \multicolumn{1}{c|}{$T$-value } & \multicolumn{1}{c|}{ Significance } \\
\hline Readability & -10.124 & 1.864 & -5.431 & $0.000^{* * *}$ \\
\hline Emotionality & 1.430 & 0.692 & 2.067 & $0.039^{*}$ \\
\hline Additional Descriptions & 16.263 & 0.688 & 23.651 & $0.000^{* * *}$ \\
\hline Question Type & -5.189 & 0.691 & -7.512 & $0.000^{* * *}$ \\
\hline Adjusted $R^{2}$ & 0.030 & & & \\
\hline Regression Model Significance & 0.000 & & & \\
\hline$F$-value & 155.253 & & & \\
\hline
\end{tabular}

Note: ${ }^{* * *}$ means $p<0.001,{ }^{* *}$ means $p<0.01,{ }^{*}$ means $p<0.05$

Question type has a significant positive impact on number of answers (coefficient $=2.406>$ $0, p<=0.001$ ), indicating that conversational questions coded as " 1 " receive more answers than informational questions coded as " 0 ". Therefore, Hypothesis $\mathrm{H} 4 \mathrm{a}$ is validated.

In terms of the degree of influence under the conditions of this study, when other independent variables remain unchanged, the number of answers potentially increases by 2.847 with the normalized readability value increasing by 1 unit; questions with additional descriptions potentially lead to 1.319 answers fewer than those without additional descriptions; conversational questions potentially result in 2.406 more answers than informational questions.

\subsection{Regression Analysis on Average Answer Length}

Using average length of answer as the dependent variable, the corresponding regression model is constructed as in Equation (5), where $A A L$ stands for Average Answer Length, $R E_{2}$ stands for Readability, $E M_{2}$ stands for Emotionality, $A D_{2}$ stands for Additional Descriptions, and $Q T_{2}$ stands for Question Type. The results of the regression analysis are summarized in Table 6.

$\hat{y}_{2}(\mathrm{AAL})=b_{20}+b_{21} \times \mathrm{RE}_{2}+b_{22} \times \mathrm{EM}_{2}+b_{23} \times \mathrm{AD}_{2}+b_{24} \times \mathrm{QT}_{2}$

The results in Table 6 indicate that this Multiple Linear Regression model pertaining to average length of answers is statistically significant $(F=155.253, p<0.001)$. In addition, all four independent variables included in the research model of this study have a statistically significant impact on average length of answers $(p<0.05)$.

Question readability has a significant negative impact on average length of answers (coefficient $=-10.124<0, p<=0.001$ ), suggesting that the greater the readability of a question, the shorter the average length of its answers, thus confirming the opposite of $H 1 b$.

Question emotionality has a significant positive impact on average length of answers (coefficient $=1.430>0, p<=0.05$ ), indicating that on average, questions with emotionality receive longer answer than questions without emotionality. Thus, Hypothesis $H 2 b$ is validated.

Additional descriptions have a significant positive impact on average length of answers (coefficient $=16.263>0, p<=0.001$ ), indicating that on average, questions with additional descriptions receive longer answers than questions without additional descriptions. Thus, Hypothesis $H 3 b$ is validated.

Question type has a significant negative impact on average length of answers (coefficient $=$ $-5.189<0, p<=0.001$ ), suggesting that on average, informational questions coded as " 0 " receive longer answers than conversational questions coded as " 1 ". Therefore, Hypothesis $H 4 b$ is validated. 
Table 7. Regression Results with Avg. Number of Likes as Dependent Variable

\begin{tabular}{|l|l|l|l|l|}
\hline \multicolumn{1}{|c|}{ Name of Variable } & \multicolumn{1}{c|}{ Coefficient } & \multicolumn{1}{c|}{$\begin{array}{c}\text { Std. } \\
\text { Deviation }\end{array}$} & \multicolumn{1}{|c|}{$T$-value } & \multicolumn{1}{c|}{ Significance } \\
\hline Readability & -0.595 & 0.031 & -19.340 & $0.000^{* * *}$ \\
\hline Emotionality & 0.010 & 0.011 & 0.838 & 0.402 \\
\hline Additional Descriptions & 0.069 & 0.011 & 6.055 & $0.000^{* * *}$ \\
\hline Question Type & 0.111 & 0.011 & 9.718 & $0.000^{* * *}$ \\
\hline Adjusted $R^{2}$ & 0.025 & & & \\
\hline Regression Model Significance & 0.000 & & & \\
\hline$F$-value & 131.414 & & & \\
\hline
\end{tabular}

Note: ${ }^{* * *}$ means $p<0.001,{ }^{* *}$ means $p<0.01,{ }^{*}$ means $p<0.05$

In terms of the degree of influence under the conditions of this study, when other independent variables remain unchanged, the average answer length potentially decreases by 10.124 with the normalized readability value increasing by 1 unit; the average answer length for questions with emotionality is 1.43 longer than those without emotionality; the average answer length of questions with additional descriptions is 16.263 longer than those without additional descriptions; the average answer length of conversational questions is 5.189 shorter than informational questions.

\subsection{Regression Analysis on Average Number of Likes}

Using average number of "likes" received by answers as the dependent variable, the regression model is constructed as in Equation (6), where $A N L$ stands for Average Number of Likes, $R E_{3}$ stands for Readability, $E M_{3}$ stands for Emotionality, $A D_{3}$ stands for Additional Descriptions, and $Q T_{3}$ stands for Question Type. The results of the analysis are shown in Table 7.

$\hat{y}_{3}(\mathrm{ANL})=b_{30}+b_{31} \times \mathrm{RE}_{3}+b_{32} \times \mathrm{EM}_{3}+b_{33} \times \mathrm{AD}_{3}+b_{34} \times \mathrm{QT}_{3}$

Results in Table 7 indicate that this Multiple Linear Regression model pertaining to average number of "likes" received by answers is statistically significant $(F=131.414, p<0.001)$ and that three of the four independent variables in the research model of this study, question readability, additional descriptions, and question type, have a statistically significant impact on average number of "likes" $(p<0.05)$.

Question readability has a significant negative impact on average number of "likes" received by a question's answers (coefficient $=-0.595<0, p<=0.001$ ), suggesting that the greater the readability of a question, the less "likes" its answers receive. Thus, the opposite of Hypothesis Hlc is confirmed.

Question emotionality has no significant impact on average number of "likes" received by its answers $(p=0.402)$, suggesting that Hypothesis $H 2 c$ is invalid.

Additional descriptions have a significant positive impact on average number of likes (coefficient $=0.069>0, \mathrm{p}<=0.001$ ), indicating that on average, answers to questions with supplemental descriptions receive more "likes" than answers to questions without supplemental descriptions. Therefore, Hypothesis $H 3 c$ is validated.

Question type has a significant positive impact on average number of "likes" (coefficient $=0.111$ $>0, p<=0.001$ ), suggesting that on average, answers to conversational questions receive more "likes" than answers to informational questions. Thus, Hypothesis $H 4 c$ is invalidated.

In terms of the degree of influence under the conditions of this study, when other independent variables remain unchanged, the average number of "likes" potentially decreases by 0.595 with 
Figure 3. Results of Hypothesis Tests

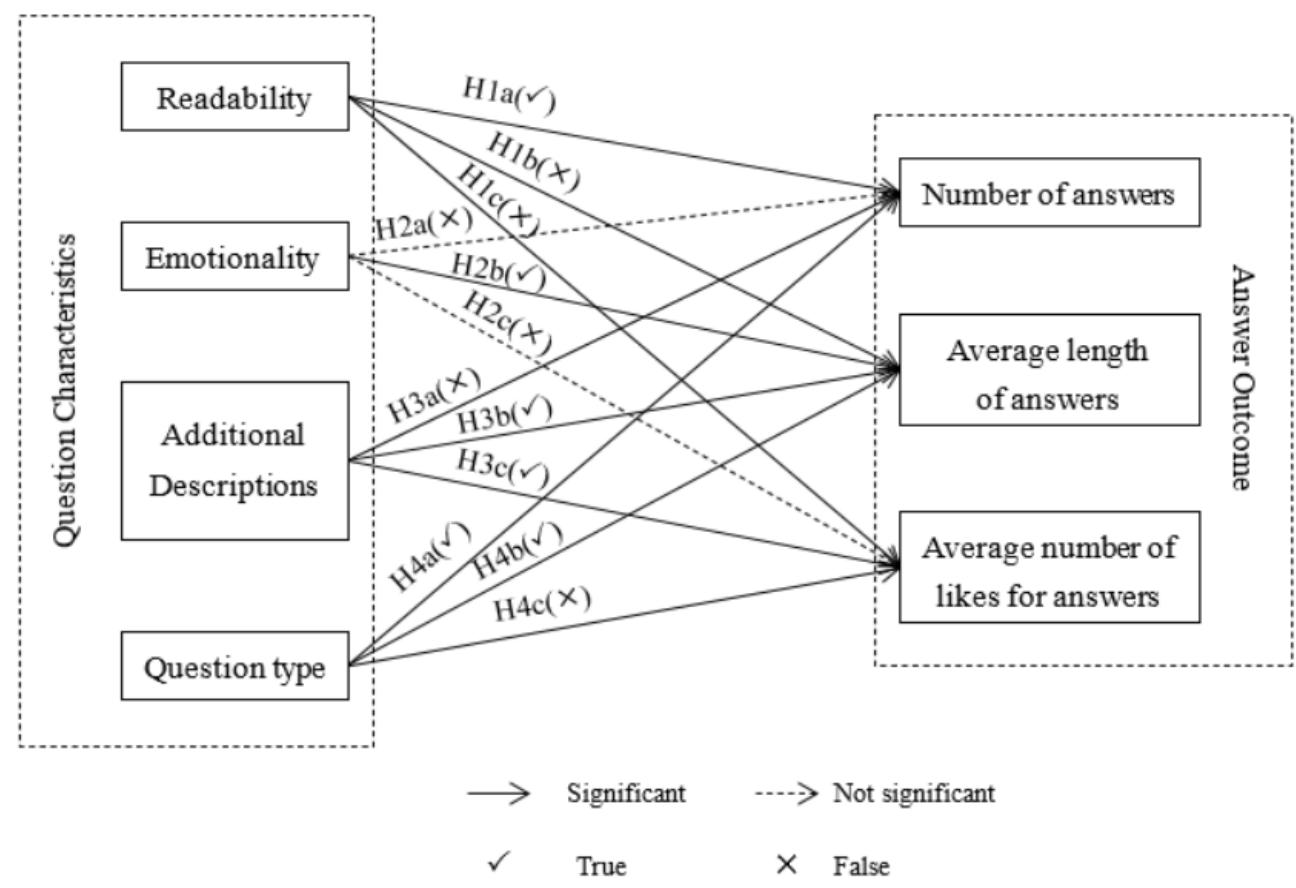

normalized readability value increasing by 1 unit; the answers to questions with additional descriptions potentially lead to 0.069 more "likes" on average than answers to questions without additional descriptions; the answers to conversational questions potentially generate 0.111 more "likes" on average than answers to informational questions.

\subsection{Result Analysis and Discussion}

Based on the results of Multiple Linear Regression analyses, the results of hypothesis tests pertaining to our research model are summarized in Figure 3.

Three of the four question characteristics in the research model of this study, question readability, additional descriptions, and question type, have significant impact on both the "quantity" aspect and the "quality" aspect of answer outcome, while question emotionality only affects the average length of answers.

\subsubsection{Impact of Question Readability on Answer Outcome}

This study reveals that question readability positively influences number of answers the question receives. In other words, the greater a question's readability, the more answers it receives. This is understandable. The readability level affects both the speed at which readers understand the question and their perceived value of answering the question. Therefore, the more readable a question is, the more likely it would attract readers' attention.

Interestingly, this study finds that question readability negatively impacts the average length of answers the question receives. In other words, the more readable a question, the shorter answers it receives. It is possible that as the readers understand the questions more thoroughly, there is a lesser need for the readers to second-guess and to "beat around the bush," hence the shorter answers.

Finally, this study finds that question readability negatively impacts the number of "likes" the question's answers receive. In other words, the more readable a question is, the less "likes" its answers 
receive. A "like" by readers other than the questioner typically expresses an agreement. As discussed already, more readable questions result in shorter answers, and shorter answers tend to be more factual and less subjective, therefore less inviting to other readers to "agree" to.

\subsubsection{Impact of Question Emotionality on Answer Outcome}

This study finds that question emotionality positively impacts average length of answers, but it has no significant impact on number of answers or on number of "likes" received by the question's answers. In other words, emotional expressions in question text increase the average length of answers the question receives, but makes no significant difference in number of the answers or in number of "likes" received by the answers.

Understandably, whether a question can attract responses from users of SAQ websites is most likely determined by the clarity of the question expression and the users' ability to answer the question. The presence of emotionality in a question neither adds to the clarity of the question nor contributes to one's ability to answer the question and is therefore not so useful in attracting bystanders to respond to the question.

However, for those users who choose to answer a specific question, emotional expressions in the question text will likely spark feelings of empathy or sympathy, causing the respondent to provide longer answers which may include emotional expressions likewise.

As mentioned earlier, a "like" by readers other than the questioner typically expresses an agreement. In deciding whether they agree to a particular answer, users on SQA websites would likely consider the information value of the answer rather than emotional expressions in the original question statement.

\subsubsection{Impact of Additional Descriptions on Answer Outcome}

This study finds that supplemental descriptions in question texts impact both the quantity and the quality aspects of answer outcome. Questions with such additional descriptions will generate fewer answers, but will generate longer answers and result in more "likes" of the answers.

Generally, questions with supplemental descriptions are more complex and seem more intimidating to average readers. Therefore, fewer people will choose to respond to them.This finding agrees with the finding of Yang et al. (2011) that complex questions do not obtain answers easily.

On the other hand, like essay quesitons on exams, answers to complex questions usually require more wording to be clear and thorough. Readers who choose to respond to such complex questions would want to fully express themselves through longer answers.

Like answers to essay questions on exams, answers to questions with additional descritptions or "complex questions" will likely contain the respondents' subjective views or opions, providing a larger ground for others to "agree" or "disagree" upon. Hence, questions with additional descriptions generate more "likes" to their answers than questions without additional descriptions.

\subsubsection{Impact of Question Type on Answer Outcome}

Harper et al. (2008) previously concluded that question type has no effect on number of answers. Our study, however, finds that question type does impact the number of answers a question receives. Specifically, we find that conversational questions generate more answers than informational questions. This finding agrees with the findings of Adamic et al. (2008) that fact-based questions tend to yield fewer answers than discussion-based questions. This is very understandable, when one asks a factbased informational question, unless the first respondent provides an inaccurate answer, others will very unlikely repeat the answer. On the other hand, a discussion-generating conversational question could induce many more responses.

This study also finds that the average answer length for informational questions is longer than for conversational questions, which somewhat differs from the finding of Harper et al. (2008). They found that questions seeking advices obtained answers nearly twice as long as factual-type questions. It is 
possible that on SQA websites, many factual-type informational questions often ask for solutions to problems, and respondents often provide lengthier answers hoping to clearly communicate instructions and solutions otherwise. On the other hand, respondents responding to opinion-based conversational questions may feel no need to provide lengthy answers.

Contrary to our hypothesis, this study finds that on average, answers to informational questions receive fewer "likes" than answers to conversational questions. Again, a "like" is an expression of agreement. Factual-type informational questions typically generate fact-based objective answers which do not easily generate "internal echoes" in others. On the other hand, answers to discussionpromoting conversational questions are typically opinion-based subjective views or feelings, which more easily generate "internal echoes" in others.

\section{CONCLUSION}

The originality of the study lies in that mixed metrics were used to measure answer outcome of a question, including the number of answers to a question, average length of answers to a question, and average number of "likes" received by answers to a question.

Prior studies have examined external influence factors of answer outcome, such as user characteristics, community culture, and incentives of the SQA websites (Jin et al., 2015; Liu \& Jansen, 2017). They have revealed potential impact of external factors on answer outcome. This study has taken a different approach and examined potential impact of question characteristics themselves on answer outcome on SQA websites (namely, question readability, question emotionality, additional descriptions, and question type). The findings of this study have enlarged the theoretical ground of answer-outcome research and have revealed that in addition to external factors, internal factors could also have significant impact on the quality of answer outcome on SQA websites. When exploring impact factors, future researchers should examine both internal and external factors.

Using Yahoo!Answers as the data source and applying Multiple Linear Regression analyses to the sample data, this study has discovered that:

(1) The readability of a question has a positive impact on the number of answers it attracts, a negative impact on the average length of its answers, and a negative impact on the average number of "likes" its answers receive.

(2) The emotionality of a question leads to longer answers but has no significant impact on the number of answers it receives or on the number of "likes" its answers receive.

(3) Additional descriptions in a question result in fewer answers to the question but longer answers and more "likes" of its answers.

(4) Conversational questions generate more answers and more "likes" of their answers than informational questions; but informational questions produce longer answers than conversational questions do.

Based on these findings, the following insights can be provided to SQA websites builders as they instruct website users on question construction. These insights can also help SQA website users build more effective questions so as to attract more and higher-quality answerers to their questions:

(1) Simple vocabulary and simple sentence structure should be used in question construction to make questions easily understandable.

(2) Whenever appropriate, it helps to include some personal emotion in the question statement to inspire potential respondents so as to obtain longer or more-detailed answers.

(3) For complex questions, present the questions using concise and plain statements first and then provide detailed clarification about the questions. 
(4) When constructing conversational questions, questioners should motivate others to participate in discussions by reducing knowledge requirements or subject constraints. When constructing informational questions, questioners should motivate higher-quality answers by clearly specifying what is sought for (such as who, what, how, and when).

One limitation of this study is possible data bias. This study utilizes only one month of questions and answers from Yahoo! Answers.

For future studies, data collection can be expanded to include multiple SQA websites and longer periods of time, and additional textual features as well as non-textual features can be included in the research model.

\section{ACKNOWLEDGMENT}

Yiming Zhao and Linrong Wu are supported by the National Natural Science Foundation of China under Grants 71874130, 71921002, and 71420107026. They are partly supported by the Ministry of Education of the People's Republic of China under Grant 18YJC870026 and by the Science Foundation of Hubei Province under Grant 2019CFA025. 


\section{REFERENCES}

Adamic, L. A., Zhang, J., Bakshy, E., \& Ackerman, M. S. (2008). Knowledge sharing and Yahoo Answers: everyone knows something. In Proceedings of the 17th International Conference on World Wide Web (WWW 2008). ACM. doi:10.1145/1367497.1367587

Agichtein, E., Castillo, C., Donato, D., Gionis, A., \& Mishne, G. (2008). Finding high-quality content in social media. Proceedings of the 2008 International Conference on Web Search and Web Data Mining (WSDM 2008), 183-194.

Alexa. (2020). The top ranked sites in references category. Retrieved April 13th, 2020, from http://www.alexa. com/topsites/ category/Top/Reference

Asaduzzaman, M., Mashiyat, A. S., Roy, C. K., \& Schneider, K. A. (2013). Answering questions about unanswered questions of Stack Overflow. In Proceedings of the 10th IEEE Working Conference on Mining Software Repositories, (pp. 97-100). IEEE. doi:10.1109/MSR.2013.6624015

Buntrock, R. E. (2007). Information and emotion: The emergent affective paradigm in information behavior research and theory. Library Management, 64(3), 1137-1142.

Cabrera, E. F., \& Cabrera, A. (2005). Fostering knowledge sharing through people management practices. International Journal of Human Resource Management, 16(5), 720-735. doi:10.1080/09585190500083020

Caprio, D. D., Santos-Arteaga, F. J., \& Tavana, M. (2015). Technology development through knowledge assimilation and innovation: A European perspective. Journal of Global Information Management, 23(2), 48-93. doi:10.4018/JGIM.2015040103

Casalo, L. V., Flavian, C., \& Cuinaliu, M. (2010). Relationship quality, community promotion and brand loyalty in virtual communities: Evidence from free software communities. International Journal of Information Management, 30(4), 357-367. doi:10.1016/j.ijinfomgt.2010.01.004

Chatterjee, S., Kar, A. K., \& Gupta, M. P. (2017). Critical success factors to establish 5G network in smart cities: Inputs for security and privacy. Journal of Global Information Management, 25(2), 15-37. doi:10.4018/ JGIM.2017040102

Chen, I. Y. L. (2007). The factors influencing members' continuance intentions in professional virtual communities - a longitudinal study. Journal of Information Science, 33(4), 451-467. doi:10.1177/0165551506075323

Choi, E., Kitzie, V., \& Shah, C. (2013, February). A machine learning-based approach to predicting success of questions on social question-answering. Proceedings of Iconference.

Choudhury, S., Alani, H., \& Kmi, U. (2014). Exploring user behavior and needs in Q\&A communities. Proceedings of the European Conference on Social Media, 80-89.

Chua, A. Y. K., \& Banerjee, S. (2015). Answers or no answers: Studying question answerability in Stack Overflow. Journal of Information Science, 41(5), 720-731. doi:10.1177/0165551515590096

Coleman, M., \& Liau, T. L. (1975). A computer readability formula designed for machine scoring. The Journal of Applied Psychology, 60(2), 283-284. doi:10.1037/h0076540

Elalfy, D., Gad, W., \& Ismail, R. M. (2017). A hybrid model to predict best answers in question answering communities. Egyptian Informatics Journal, 19(1), 21-31. doi:10.1016/j.eij.2017.06.002

Flesch, R. (1948). A new readability yardstick. The Journal of Applied Psychology, 32(3), 221-233. doi:10.1037/ h0057532 PMID: 18867058

Gazan, R. (2014). Social Q\&A. Journal of the Association for Information Science and Technology, 62(12), 2301-2312.

Gunning, R. (1968). Technique of clear writing. McGraw-Hill.

Harper, F. M., Moy, D., \& Konstan, J. A. (2009). Facts or friends: distinguishing informational and conversational questions in SQA sites. In Proceedings of the 27th Sigchi Conference on Human Factors in Computing Systems. ACM. doi:10.1145/1518701.1518819 
Harper, F. M., Raban, D. R., Rafaeli, S., \& Konstan, J. A. (2008). Predictors of answer quality in online Q\&A sites. In Proceedings of the 26th Annual SIGCHI Conference on Human Factors in Computing Systems, (pp. 865-874). DBLP. doi:10.1145/1357054.1357191

Harper, F. M., Weinberg, J., Logie, J., \& Konstan, J. A. (2010). Question types in SQA sites. First Monday, 15(7).

Huang, W., Le, T., Li, X., \& Gandha, L. (2006). Categorizing Web features and functions to evaluate commercial websites: An assessment framework and an empirical investigation of Australian companies. Industrial Management \& Data Systems, 106(4), 523-539. doi:10.1108/02635570610661606

Hung, S. Y., Huang, W. M., Yen, D. C., Chang, S. I., \& Lu, C. C. (2016). Effect of information service competence and contextual factors on the effectiveness of strategic information systems planning in hospitals. Journal of Global Information Management, 24(1), 14-36. doi:10.4018/JGIM.2016010102

Jeon, J., Croft, W. B., Lee, J. H., \& Park, S. (2006, August). A framework to predict the quality of answers with non-textual features. Proceedings of the 29th annual international ACM SIGIR conference on Research and development in information retrieval, 228-235. doi:10.1145/1148170.1148212

Jiang, T., Zhang, C., Li, Z., Fan, C., \& Yang, J. (2018). Information encountering on SQA sites: a diary study of the process. In G. Chowdhury, J. McLeod, V. Gillet, \& P. Willett (Eds.), Lecture Notes in Computer Science: Vol. 10766. Transforming Digital Worlds. iConference 2018. Springer.

Jin, J., Li, Y., Zhong, X., \& Zhai, L. (2015). Why users contribute knowledge to online communities: An empirical study of an online SQA community. Information \& Management, 52(7), 840-849. doi:10.1016/j.im.2015.07.005

Kenton, W. (2019, April). Multiple Linear Regression-MLR Definition. Retrieved from https://www.investopedia. com $/$ terms $/ \mathrm{m} / \mathrm{mlr}$.asp

Kim, S., \& Oh, S. (2009). Users' relevance criteria for evaluating answers in an SQA site. Journal of the American Society for Information Science and Technology, 60(4), 716-727. doi:10.1002/asi.21026

Kincaid, E. H. (1992). The Medicare program: Exploring federal health care policy. North Carolina Medical Journal, 53(11), 596-601. PMID:1436155

Klare, G. R. (1974). Assessing readability. Reading Research Quarterly, 10(1), 62-102. doi:10.2307/747086

Lane, V. R., Khuntia, J., Parthasarathy, M., \& Hazarika, B. B. (2017). The impact of the Internet on values in India: Shifts in self-enhancement and self-transcendence amongst Indian youth. Journal of Global Information Management, 25(3), 98-120. doi:10.4018/JGIM.2017070106

Li, L., He, D., Jeng, W., Goodwin, S., \& Zhang, C. (2015). Answer quality characteristics and prediction on an academic Q\&A Site: A case study on ResearchGate. In Proceedings of the 24th International Conference on World Wide Web, (pp. 1453-1458). ACM. doi:10.1145/2740908.2742129

Li, Z., Detlor, B., \& Connelly, C. E. (2016). Sharing knowledge in SQA sites: The unintended consequences of extrinsic motivation. Journal of Management Information Systems, 33(1), 70-100. doi:10.1080/07421222. 2016.1172459

Liu, Z., \& Jansen, B. J. (2017). Identifying and predicting the desire to help in social question and answering. Information Processing \& Management, 53(2), 490-504. doi:10.1016/j.ipm.2016.05.001

McLaughlin, G. H. (1969). SMOG grading-a new readability formula. Journal of Reading, 12(8), 639-646.

Oh, S. (2012). The characteristics and motivations of health answerers for sharing information, knowledge, and experiences in online environments. Journal of the American Society for Information Science and Technology, 63(3), 543-557. doi:10.1002/asi.21676

Oh, S. (2018). SQA. In P. Brusilovsky \& D. He (Eds.), Social Information Access (pp. 75-107). Springer, Cham. doi:10.1007/978-3-319-90092-6_3

Ren, R., Duan, H., Liu, W., \& Liu, J. (2018) AUnet: an unsupervised method for answer reliability evaluation in community QA systems. In Proceedings of Asia-Pacific Web (APWeb) and Web-Age Information Management (WAIM) Joint International Conference on Web and Big Data, (pp. 281-292). Springer. doi:10.1007/978-3030-01298-4_24 
Roztocki, N., \& Weistroffer, H. R. (2011). Information technology success factors and models in developing and emerging economies. Information Technology for Development, 17(3), 163-167. doi:10.1080/02681102. 2011.568220

Saha, R. K., Saha, A. K., \& Perry, D. E. (2013, August). Toward understanding the causes of unanswered questions in software information sites: a case study of Stack Overflow. In Proceedings of the 2013 9th Joint Meeting on Foundations of Software Engineering, (pp. 663-666). ACM. doi:10.1145/2491411.2494585

Scott, B. (2019). The Automated Readability Index (ARI). Retrieved September 13th, 2020, from https://www. readabilityformulas.com/automated-readability-index.php

Shah, C., \& Pomerantz, J. (2010, July). Evaluating and predicting answer quality in community QA. In Proceedings the 33rd International ACM SIGIR Conference on Research and Development in Information Retrieval. ACM. doi: $10.1145 / 1835449.1835518$

Shah, C., Radford, M. L., Connaway, L. S., Choi, E., \& Kitzie, V. (2012). "How much change do you get from $40 \$$ ?" - analyzing and addressing failed questions on SQA. Proceedings of the Association for Information Science and Technology, 49(1), 1-10. doi:10.1002/pra2.2015.145052010051

Silic, M., \& Back, A. (2016). What are the keys to a successful mobile payment system? case of Cytizi: Mobile payment system. Journal of Global Information Management, 24(3), 1-20. doi:10.4018/JGIM.2016070101

Sin, S. C. J., Lee, C. S., \& Chen, X. (2018, November). Rewarding, but not for everyone: interaction acts and perceived post quality on social Q\&A sites. Proceedings of the 20th International Conference on Asia-Pacific Digital Libraries (ICADL 2018). doi:10.1007/978-3-030-04257-8_12

Smith, E. A., \& Senter, R. J. (1967). Automated readability index. Aerospace Medical Research Laboratories.

Soja, P. (2016). Reexamining critical success factors for enterprise system adoption in transition economies: Learning from Polish adopters. Information Technology for Development, 22(2), 279-305. doi:10.1080/0268 1102.2015.1075189

Steven, L. (2019, October). TextBlob: Simplified text processing. Retrieved from https://textblob.readthedocs. io/en/dev/

Teevan, J., Morris, M. R., \& Panovich, K. (2011). Factors affecting response quantity, quality, and speed for questions asked via social network status messages. Proceedings of Fifth International AAAI Conference on Weblogs and Social Media.

Teo, T. S. H. (2007). Organizational characteristics, modes of Internet adoption and their impact: A Singapore perspective. Journal of Global Information Management, 15(2), 91-117. doi:10.4018/jgim.2007040104

Wang, S., Chen, T., \& Hassan, A. E. (2018). Understanding the factors for fast answers in technical Q\&A websites. Empirical Software Engineering, 23(3), 1552-1593. doi:10.1007/s10664-017-9558-5

Wasko, M. M., \& Faraj, S. (2000). It is what one does: Why people participate and help others in electronic communities of practice. The Journal of Strategic Information Systems, 9(2-3), 155-173. doi:10.1016/S09638687(00)00045-7

Wasko, M. M., \& Faraj, S. (2005). Why should I share? examining social capital and knowledge contribution in electronic networks of practice. Management Information Systems Quarterly, 29(1), 35-57. doi:10.2307/25148667

Yahoo Research. (2019). Questions on Yahoo Answers labeled as either informational or conversational. Retrieved from https://webscope.sandbox.yahoo.com/catalog.php?datatype $=1 \& \mathrm{did}=82$

Yang, L., Bao, S., Lin, Q., Wu, X., Han, D., \& Su, Z. (2011). Analyzing and predicting not-answered questions in community-based question answering services. In Proceedings of the Association for the Advancement of Artificial Intelligence conference, (pp. 1273-1278). AAAI.

Zhang, J., Cai, X., Le, T., Fei, W., \& Ma, F. (2019). A study on effective measurement of search results from search engines. Journal of Global Information Management, 27(1), 196-221. doi:10.4018/JGIM.2019010110

Zhang, J., Chen, Y., Zhao, Y., Wolfram, D., \& Ma, F. (2020). Public health and social media: A study of Zika virus-related posts on Yahoo! Answers. Journal of the Association for Information Science and Technology, 71(3), 282-299. doi:10.1002/asi.24245 
Zhang, J., \& Zhao, Y. (2013). A user term visualization analysis based on a social question and answer log. Information Processing \& Management, 49(3), 1019-1048. doi:10.1016/j.ipm.2013.04.003

Zhang, J., Zhao, Y., Cai, X., Le, T., Fei, W., \& Ma, F. (2020). A Comparison of retrieval result relevance judgments between American and Chinese users. Journal of Global Information Management, 28(3), 148-168. doi:10.4018/JGIM.2020070108

Zhao, Y., Chen, B., Zhang, J., Ding, Y., Mao, J., \& Zhou, L. (2018). An investigation on the evolution of diabetes data in social Q\&A logs. Data and Information Management, 2(1), 37-48. doi:10.2478/dim-2018-0002

Zhao, Y., Zhang, J., Xia, X., \& Le, T. (2019). Evaluation of Google question-answering quality. Library Hi Tech, 37(2), 312-328. doi:10.1108/LHT-10-2017-0218

Yiming Zhao is an associate professor at the School of Information Management, Wuhan University, China. He is Director of Data Analysis and Information Resource Planning Laboratory in the Center for Studies of Information Resources, Wuhan University, China. His research interests include text analysis, information retrieval, and information-seeking behavior.

Linrong Wu is a graduate student at the School of Information Management, Wuhan University, China. His research interest is text analysis.

Jin Zhang is a full professor at the School of Information Studies, University of Wisconsin-Milwaukee, U.S.A. He has published papers in journals such as Journal of the American Society for Information Science and Technology, Information Processing \& Management, Journal of Documentation, Journal of Intelligent Information Systems, Online Information Review, etc. His book "Visualization for Information Retrieval" was published in the Information Retrieval Series by Springer in 2008. His research interests include visualization for information retrieval, information retrieval algorithm, metadata, search engine evaluation, consumer health informatics, social media, social network analysis, data mining, knowledge system evaluation, and human computer interface design.

Taowen Le is a tenured professor and former department chair of information systems \& technologies at Weber State University, Business Division Chair of Utah Academy of Science, Arts, and Letters. 